\title{
Comparative Effectiveness of Different Types of Cervical Laminoplasty
}

\author{
John G. Heller ${ }^{1}$ Annie L. Raich ${ }^{2}$ Joseph R. Dettori ${ }^{2} \quad$ K. Daniel Riew ${ }^{3}$ \\ ${ }^{1}$ Department of Orthopaedic Surgery, Emory Spine Center, Atlanta, \\ Georgia, United States \\ 2 Spectrum Research, Inc., Tacoma, Washington, United States \\ ${ }^{3}$ Department of Orthopedic Surgery, Washington University, St. Louis, \\ Missouri, United States

\begin{abstract}
Address for correspondence John G. Heller, MD, Department of Orthopaedic Surgery, Emory Spine Center, 59 Executive Park South, Suite 2000, Atlanta, GA 30329, United States (e-mail: john.heller@emoryhealthcare.org).
\end{abstract}

Evid Based Spine Care J 2013;4:105-115.

\author{
Abstract

\section{Keywords} \\ - laminoplasty \\ - cervical myelopathy \\ - cervical spondylotic \\ myelopathy \\ - OPLL \\ - open door \\ laminoplasty \\ - french door \\ laminoplasty \\ - miniplates \\ - complications
}

Study Design Systematic review.

Study Rationale Numerous cervical laminoplasty techniques have been described but there are few studies that have compared these to determine the superiority of one over another.

Clinical Questions The clinical questions include key question (KQ)1: In adults with cervical myelopathy from ossification of the posterior longitudinal ligament (OPLL) or spondylosis, what is the comparative effectiveness of open door cervical laminoplasty versus French door cervical laminoplasty? KQ2: In adults with cervical myelopathy from OPLL or spondylosis, are postoperative complications, including pain and infection, different for the use of miniplates versus the use of no plates following laminoplasty? KQ3: Do these results vary based on early active postoperative cervical motion?

Materials and Methods A systematic review of the English-language literature was undertaken for articles published between 1970 and March 11, 2013. Electronic databases and reference lists of key articles were searched to identify studies evaluating (1) open door cervical laminoplasty and French door cervical laminoplasty and (2) the use of miniplates or no plates in cervical laminoplasty for the treatment of cervical spondylotic myelopathy or OPLL in adults. Studies involving traumatic onset, cervical fracture, infection, deformity, or neoplasms were excluded, as were noncomparative studies. Two independent reviewers (A.L.R., J.R.D.) assessed the level of evidence quality using the Grades of Recommendations Assessment, Development and Evaluation system, and disagreements were resolved by consensus.

Results We identified three studies (one of class of evidence [CoE] II and two of CoE III) meeting our inclusion criteria comparing open door cervical laminoplasty with French door laminoplasty and two studies (one CoE II and one CoE III) comparing the use of miniplates with no plates. Data from one randomized controlled trial (RCT) and two retrospective cohort studies suggest no difference between treatment groups regarding improvement in myelopathy. One RCT reported significant improvement in axial pain and significantly higher short-form 36 scores in the French door laminoplasty treatment group. Overall, complications appear to be higher in the open door group than the French door group, although complete reporting of complications was poor in received

March 28, 2013

accepted after revision

July 18, 2013 (c) 2013 Georg Thieme Verlag KG

Stuttgart · New York
DOI http://dx.doi.org/ 10.1055/s-0033-1357361. ISSN 1663-7976. 
all studies. Overall, data from one RCT and one retrospective cohort study suggest that the incidence of complications (including reoperation, radiculopathy, and infection) is higher in the no plate treatment group compared with the miniplate group. One RCT reported greater pain as measured by the visual analog scale score in the no plate treatment group. There was no evidence available to assess the effect of early cervical motion for open door cervical laminoplasty compared with French door laminoplasty. Both studies comparing the use of miniplates and no plates reported early postoperative motion. Evidence from one RCT suggests that earlier postoperative cervical motion might reduce pain.

Conclusion Data from three comparative studies are not sufficient to support the superiority of open door cervical laminoplasty or French door cervical laminoplasty. Data from two comparative studies are not sufficient to support the superiority of the use of miniplates or no plates following cervical laminoplasty. The overall strength of evidence to support any conclusions is low or insufficient. Thus, the debate continues while opportunity exists for the spine surgery community to resolve these issues with appropriately designed clinical studies.

\section{Study Rationale and Context}

The debate regarding the preferred surgical treatment option for cervical myelopathy and the relative indications for one procedure versus another is focused on three procedures at present: anterior decompression and fusion, posterior laminectomy and fusion, or laminoplasty. As the concept of laminoplasty evolved during the 1970s and 1980s, two competing schools of thought emerged; the so-called "open door" and "French door" methods. ${ }^{1,2}$ Discussion about the merits of one method versus the other continues, with a nearly equal prevalence of their use in Japan. Other regions of the world have tended to adopt predominantly one method, due in large part to the influence of teachers passing on the lessons of a preferred technique, rather than teaching both methods to successive generations of residents and fellows. ${ }^{3}$ Whether there is a meaningful clinical difference between the two methods of laminoplasty is unclear. Moreover, with the recent adoption of miniplate fixation of the laminae in their new position and a shift toward early active cervical range of motion during the postoperative period, it seems prudent to investigate whether there has been some incremental benefit for patients.

\section{Objective or Clinical Questions}

KQ1: In adults with cervical myelopathy from ossification of the posterior longitudinal ligament (OPLL) or spondylosis, what is the comparative effectiveness of open door cervical laminoplasty versus French door cervical laminoplasty?

KQ2: In adults with cervical myelopathy from OPLL or spondylosis, are postoperative complications, including pain and infection, different for the use of miniplates versus the use of no plates following laminoplasty?

KQ3: Do these results vary based on early active postoperative cervical motion?

\section{Materials and Methods}

Study design: Systematic review.

Search: The databases included PubMed, Cochrane, and National Guideline Clearinghouse Databases, as well as bibliographies of key articles.

Dates searched: The data were searched up through March 11, 2013.

Inclusion criteria: In patients aged 18 years or older with cervical myelopathy from cervical spondylotic myelopathy (CSM) or ossification of posterior longitudinal ligament (OPLL): Studies directly comparing open door cervical laminoplasty with French door cervical laminoplasty and the use of miniplates with no plates in cervical laminoplasty were included in the study.

Exclusion criteria: Studies in patients younger than 18 years, those with a cervical fracture, neoplasm, infection, or deformity; noncomparative studies, comparative studies with less than five patients per treatment group; nonhuman in vivo, in vitro, and biomechanical studies were excluded.

Outcomes: The outcome of the study was improvement in myelopathy (Japanese Orthopedic Association [JOA], Nurick scores), pain (visual analog scale [VAS], and complications.

Analysis: Descriptive statistics, means, standard deviation, and ranges were abstracted from the original reports as available. Means, standard deviation, and $p$ values for JOA and Nurick scores were calculated from individual patient data in one study. Pooling of data was not done because of concerns regarding study quality and heterogeneity of treatments and study populations.

Overall strength of evidence: Risk of bias determined using a class of evidence ( $\mathrm{CoE}$ ) rating scheme developed by the Oxford Centre for Evidence-based Medicine and modified for orthopedic surgery by The Journal of Bone and Joint Surgery American Volume (J Bone Joint Surg Am) $)^{4}$ (see online supplementary material). The overall body of evidence with 
respect to each key question (KQ) was determined based on modified precepts outlined by the Grades of Recommendation Assessment, Development and Evaluation (GRADE) system. ${ }^{5}$

Details about the methods can be found in the online supplementary material.

\section{Results}

From a total of 291 citations retrieved, 9 citations were evaluated for full-text review, and 5 citations met the inclusion criteria for this report (-Fig. 1). Three studies compared the use of open door cervical laminoplasty with French door cervical laminoplasty ( $\mathbf{- T a b l e ~} \mathbf{1}$ ): one randomized controlled trial (RCT) (CoE II) ${ }^{6}$ and two retrospective cohort studies (CoE III). ${ }^{7,8}$ Two studies compared the use of miniplates with no plates in open door cervical laminoplasty (-Table 2): one RCT $(\mathrm{CoE} \text { II })^{9}$ and one retrospective cohort study (CoE III). ${ }^{10}$ The study populations comprised a mixture of mostly CSM and OPLL patients. Populations in included studies were predominantly male and middle-aged patients. Mean length of follow-up ranged from 20 to 60.1 months. All of the studies reported follow-up rates greater than $80.3 \% .^{6-10}$

Further details on the CoE rating for these studies can be found in the online supplementary material.

\section{Comparative Effectiveness of Open Door versus French Door Cervical Laminoplasty}

Improvement in Myelopathy

One RCT and two retrospective cohort studies assessed neurologic improvement as measured by JOA scores or JOA recovery rate. All three studies found no significant difference between open door cervical laminoplasty and French door cervical laminoplasty treatment groups at follow-up regarding improvement in myelopathy as measured by either of

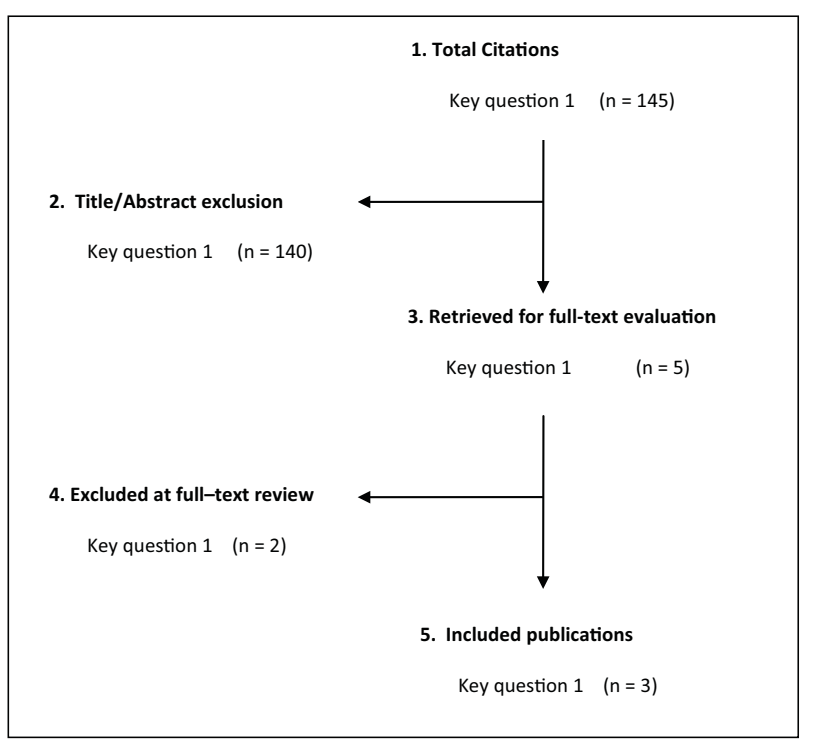

Fig. 1 Flowchart showing results of literature search. these measures (see Table 1 in online supplementary material). ${ }^{6-8}$

- Okada et $\mathrm{al}^{6}$ measured neurologic improvement using JOA scores and JOA recovery rates. This study reported that there were no significant differences between treatment groups at 26.9 months using either measure.

- Naito et $\mathrm{l}^{8}$ used the JOA recovery rate to measure change in myelopathy severity. At 60.1 months, $73 \%$ of open door patients and $79 \%$ of French door patients showed excellent or good JOA recovery rates ( $p=$ not reported [NR]).

- Yue et al ${ }^{7}$ calculated maximum and final gain in JOA scores, as well as the patients' maximum and final recovery rates. There was no significant difference between the open door and French door groups at follow-up.

\section{Pain Measured by VAS}

One study reported a significant improvement in pain in French door patients compared with open door patients at follow-up.

- One RCT (Okada et al) ${ }^{6}$ reported that French door patients showed significantly less axial pain compared with open door patients $(p<0.05)$ at 26.9 months. Although the patients in the French door treatment group experienced less pain at follow-up, open door patients experienced worse pain at follow-up (see Table 1 in online supplementary material).

Health care-Related Quality of Life Measured by SF-36 One study reported a better health care-related quality of life in French door patients compared with open door patients at follow-up.

- One $\mathrm{RCT}^{6}$ reported that all eight subscales of the short form 36 (SF-36) were equivalent preoperatively. At mean follow-up of 26.9 months, there was significantly greater improvement in the scores for bodily pain, general health, vitality, and mental health in the French door treatment group $(p<0.05)$ (see Table 1 in online supplementary material).

\section{Complications}

Overall, the incidence of complications appears to be higher in the open door laminoplasty treatment group compared with the French door group (-Table $\mathbf{3}$ ). Only one study reported overall incidence of complications, with $67 \%$ in the open door laminoplasty group versus $16 \%$ in the French door group $(p=\mathrm{NR})^{7}$

- Two studies reported reoperation rates for reasons including restenosis and a dural tear. Only one of the studies reported reoperation rates for both treatment groups: $6 \%$ for patients who received open door laminoplasty versus $0 \%$ for those who received French door laminoplasty ${ }^{6}$. Yue et $\mathrm{al}^{7}$ reported that $4 \%$ of the French door treatment group required further surgery to repair a dural tear; additional nonpreplanned surgery was performed on two patients, but the type of laminoplasty treatment was not reported.

- Various complication rates, including C7 radiculopathy (12\%), shoulder numbness/pain (12\%), C5 palsy (6\%), transient hemiparesis (6\%), and superficial wound infection 


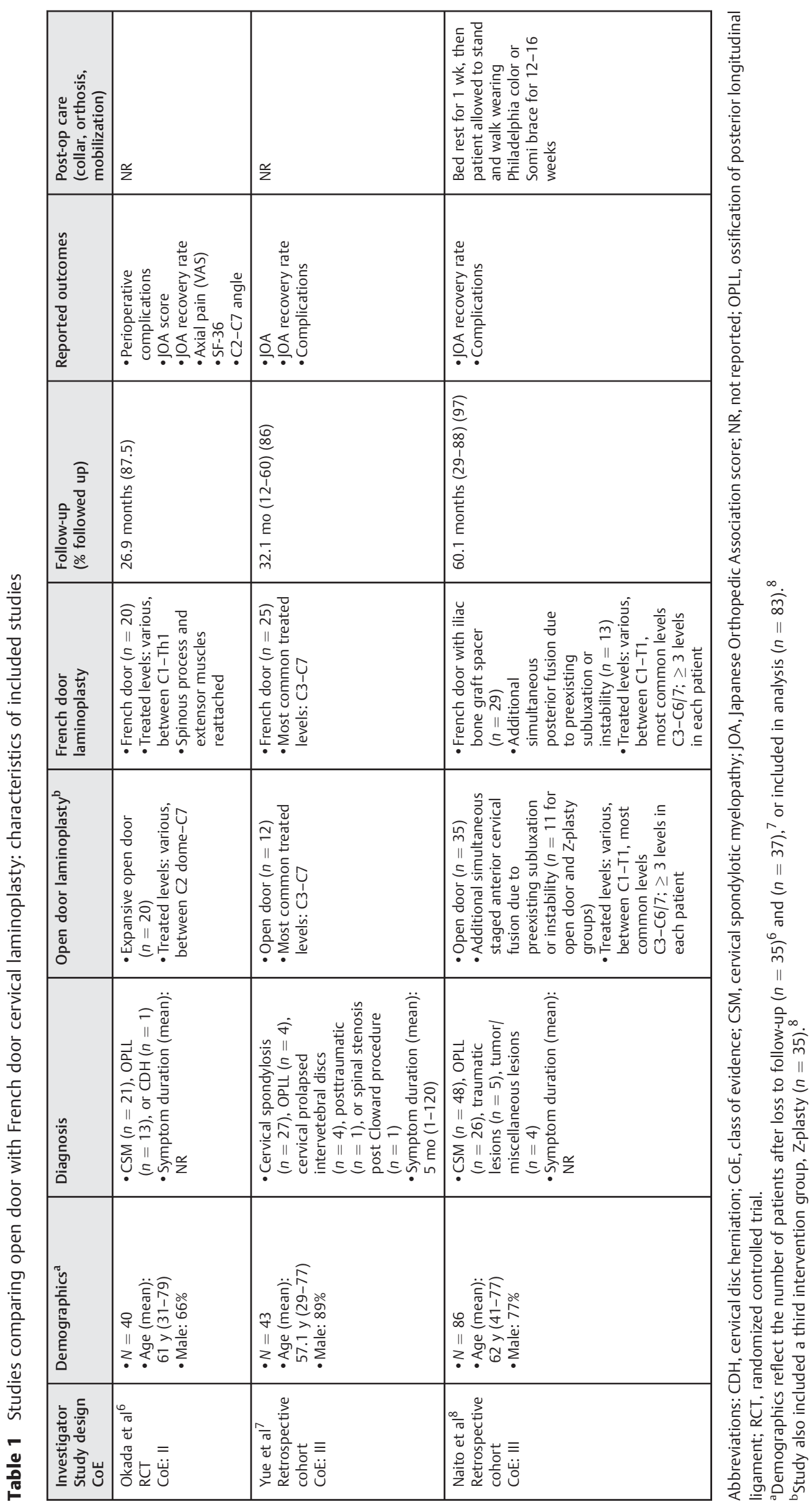




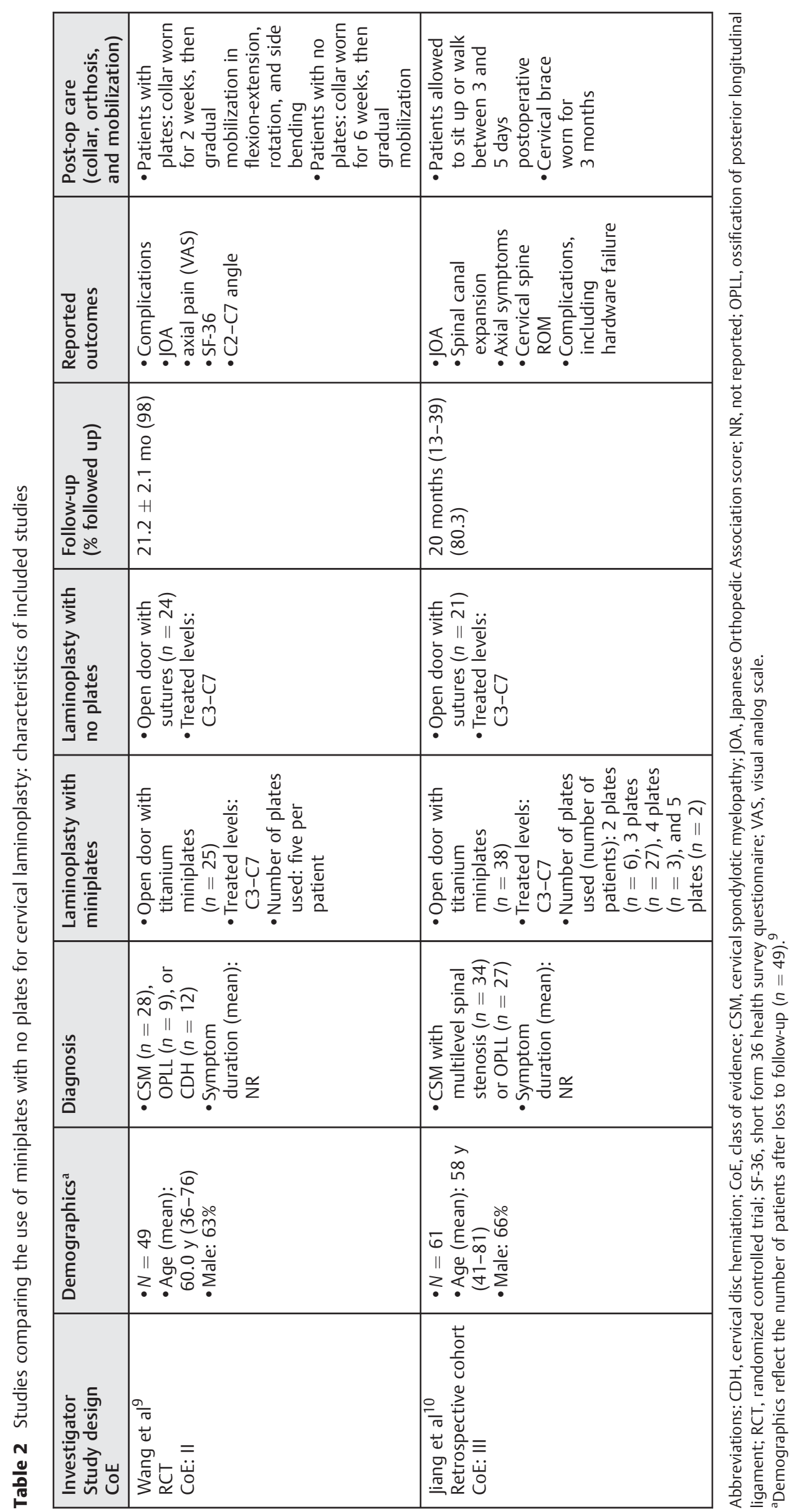


Table 3 Studies comparing open door with French door cervical laminoplasty: postoperative complication rates

\begin{tabular}{|c|c|c|}
\hline & Open door, \% & French door, \% \\
\hline \multicolumn{3}{|c|}{ Reoperation (causes) ${ }^{\mathbf{a}}$} \\
\hline Okada et $\mathrm{al}^{6}$ & $\begin{array}{l}6(1 / 17) \\
\text { Cause: spinal } \\
\text { canal restenosis } \\
(n=1)\end{array}$ & $0(0 / 18)$ \\
\hline Yue et $\mathrm{al}^{7}$ & NR & $\begin{array}{l}4(1 / 25) \\
\text { Cause: dural tear }\end{array}$ \\
\hline \multicolumn{3}{|c|}{ C7 radiculopathy } \\
\hline Okada et al ${ }^{6}$ & $12(2 / 17)$ & NR \\
\hline \multicolumn{3}{|c|}{ Shoulder numbness/pain } \\
\hline Okada et $\mathrm{al}^{6}$ & $12(2 / 17)$ & NR \\
\hline \multicolumn{3}{|c|}{ C5 palsy (transient) } \\
\hline Okada et $\mathrm{al}^{6}$ & $6(1 / 17)$ & NR \\
\hline \multicolumn{3}{|c|}{ Hemiparesis (transient) } \\
\hline Okada et $\mathrm{al}^{6}$ & $6(1 / 17)$ & NR \\
\hline \multicolumn{3}{|c|}{ Wound infection (superficial) } \\
\hline Okada et al ${ }^{6}$ & $6(1 / 17)$ & NR \\
\hline \multicolumn{3}{|c|}{ CSF leakage/dural tear (intraoperative) } \\
\hline Okada et al ${ }^{6}$ & NR & $6(1 / 18)$ \\
\hline Yue et $\mathrm{al}^{7}$ & NR & $4(1 / 25)$ \\
\hline Naito et $\mathrm{al}^{8}$ & $3(1 / 35)$ & NR \\
\hline \multicolumn{3}{|l|}{ Bleeding } \\
\hline Okada et $\mathrm{al}^{6}$ & $12(2 / 17)$ & NR \\
\hline Yue et $\mathrm{al}^{\mathrm{b}}$ & $8(1 / 12)$ & NR \\
\hline \multicolumn{3}{|c|}{ Misrecognition of surgical level } \\
\hline Okada et al ${ }^{6,7}$ & NR & $6(1 / 18)$ \\
\hline \multicolumn{3}{|l|}{ Restenosis } \\
\hline Okada et al ${ }^{6}$ & $6(1 / 17)$ & $0(0 / 18)$ \\
\hline \multicolumn{3}{|c|}{ Right facet fracture (C7/Th1) } \\
\hline Okada et al ${ }^{6}$ & $6(1 / 17)$ & NR \\
\hline
\end{tabular}

Abbreviations: CSF, cerebrospinal fluid; NR: not reported.

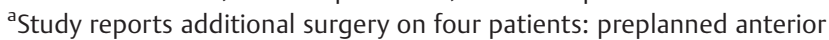
spinal fusion $(n=2)$, and anterior spinal fusion from numbness $(n=1)$ or $\mathrm{C} 5$ radiculopathy $(n=1)$; treatment group $\mathrm{NR}^{7}$; study reports reoperation (anterior cervical fusion) for two patients, but it is unclear which type of cervical laminoplasty these patients received. ${ }^{8}$

bStudy reports complications, including superficial wound infections or blood loss of $>500 \mathrm{~mL}$, in 10 patients; details, including treatment group, NR. ${ }^{7}$

(6\%) were reported in one study in the open door laminoplasty treatment group. ${ }^{6}$

- Three studies reported surgical complication rates. There was a higher rate of CSF leakage or dural tear in French door patients $(4-6 \%)^{6,7}$ compared with open door patients (3\%). ${ }^{8}$ Other reported surgical complication rates included excessive bleeding (open door patients: $8-12 \%)^{6,7}$ and misrecognition of surgical level (French door patients: $6 \%)^{6}$

- Other types of complication rates reported included: restenosis (open door suture anchor method 6\% vs. French door $0 \%)^{6}$ and a right facet fracture (open door: $\left.6 \%\right)^{6}$

\section{Effect of Early Motion}

None of the included studies conducted a formal analysis of the effect of early cervical motion on postoperative axial pain and only one study reported on postoperative care, where patients in both treatment groups received the same care. $^{8}$

\section{Comparative Safety of Miniplates versus No Plates in Cervical Laminoplasty}

Of the two studies included in this $\mathrm{KQ}$ only one study compared overall complication rates between treatment groups. Wang et $\mathrm{al}^{9}$ reported that the incidence of perioperative complications was higher in the no plate treatment group compared with the miniplate group $(p=N R)$. In both studies, the rates of reoperation, radiculopathy, infection, and restenosis were generally higher in the no plate group.

\section{Reoperation}

- One RCT (Wang et al) ${ }^{9}$ reported three patients (13\%) in the no plate treatment group requiring reoperation due to restenosis. No patients in the miniplate group required additional surgery and there were no instances of restenosis (-Table 4 ).

\section{Axial Pain Measured by VAS}

- Wang et $\mathrm{al}^{9}$ reported that patients receiving miniplates experienced significantly less pain at 21.2 months as measured by a VAS scale $(27.2 \mathrm{~mm} \pm 30.4)$ compared with patients receiving no plates $(38.8 \mathrm{~mm} \pm 30.2$, $p=0.046)$. Although the patients in the miniplate treatment group experienced less pain at follow-up than at baseline, the patients receiving no plates experienced worse pain at follow-up (see Table 2 in online supplementary material).

\section{Pain}

Both studies reported on pain, with one study reporting that the miniplate treatment group had lower rate of shoulder pain and the other study reporting that the no plate group had a lower rate of axial pain (-Table 4 ).

- One RCT (Wang et al) ${ }^{9}$ reported that one patient (4\%) in the miniplate treatment group experienced bilateral shoulder pain compared with two patients in the no plate treatment group (8\%).

- Jiang et $\mathrm{al}^{10}$ reported that 6 patients (35\%) in the no plate group experienced axial pain compared with 12 patients $(38 \%)$ in the miniplate group.

\section{Neurological Complications}

- Both studies reported on restenosis. Wang et $\mathrm{al}^{9}$ reported three cases (13\%) of restenosis in the no plate treatment 
Table 4 Studies comparing the use of miniplates with no plates in cervical laminoplasty: postoperative complication rates

\begin{tabular}{|c|c|c|c|c|}
\hline & \multicolumn{2}{|c|}{ Wang et $\mathrm{al}^{9}$} & \multicolumn{2}{|c|}{ Jiang et $\mathrm{a}^{10}$} \\
\hline & Plates, \% & No plates, \% & Plates, \% & No plates, \% \\
\hline \multicolumn{5}{|l|}{ Reoperation } \\
\hline Reoperation (causes) & $0(0 / 25)$ & $\begin{array}{l}13(3 / 24) \\
\text { Cause: restenosis }(n=3)\end{array}$ & NR & NR \\
\hline \multicolumn{5}{|l|}{ Pain } \\
\hline Axial pain & NR & NR & $38(12 / 32)$ & $35(6 / 17)$ \\
\hline Shoulder pain (bilateral) & $4(1 / 25)$ & $8(2 / 24)$ & NR & NR \\
\hline \multicolumn{5}{|l|}{ Neurological complications } \\
\hline Restenosis & $0(0 / 25)$ & $13(3 / 24)$ & $0(0 / 32)$ & $0(0 / 17)$ \\
\hline C5 radiculopathy/C5 palsy (transient) & $4(1 / 25)$ & $13(3 / 24)$ & $3(1 / 32)$ & $6(1 / 17)$ \\
\hline C7 radiculopathy & NR & $4(1 / 24)$ & NR & NR \\
\hline Numbness (right shoulder) & $4(1 / 25)$ & NR & NR & NR \\
\hline \multicolumn{5}{|l|}{ Infection } \\
\hline Wound (superficial) & $0(0 / 25)$ & NR & $6(2 / 32)$ & $12(2 / 17)$ \\
\hline \multicolumn{5}{|l|}{ Surgical complications } \\
\hline CSF leakage & $4(1 / 25)$ & $4(1 / 24)$ & NR & NR \\
\hline Spinal cord injury & $0(0 / 25)$ & $0(0 / 24)$ & NR & NR \\
\hline Bleeding & $0(0 / 25)$ & $0(0 / 24)$ & NR & NR \\
\hline \multicolumn{5}{|l|}{ Other complications } \\
\hline Cardiopulmonary event & NR & NR & NR & $6(1 / 17)$ \\
\hline Failed plates & $0(0 / 25)$ & $\mathrm{n} / \mathrm{a}$ & $0(0 / 32)$ & $\mathrm{n} / \mathrm{a}$ \\
\hline
\end{tabular}

Abbreviations: CSF, cerebrospinal fluid; n/a, not applicable; NR, not reported.

group compared with no cases in the miniplate group (0\%). Jiang et $\mathrm{al}^{10}$ reported that no patients in either treatment group experienced restenosis ( - Table 4 ).

- Wang et $\mathrm{al}^{9}$ reported one patient (4\%) in the miniplate group experiencing numbness in the right shoulder. The no plate group experienced three instances of transient $\mathrm{C} 5$ root palsy (13\%) and one temporary C7 dysesthesia (4\%) compared with one case of transient C5 root palsy (4\%) in the miniplate group.

- Jiang et al ${ }^{10}$ reported one case of transient C5 palsy in each treatment group (3\% in the miniplate treatment group, $6 \%$ in the no plate treatment group).

\section{Infection}

- Jiang et $\mathrm{al}^{10}$ reported two cases of superficial wound infections in each treatment group (6\% in the miniplate treatment group, $12 \%$ in the no plate treatment group) (-Table 4).

- Wang et $\mathrm{al}^{9}$ reported that there were no cases of infection in the miniplate group and provided no information for the no plate group.

\section{Surgical Complications}

- One study (Jiang et al) ${ }^{10}$ reported one case of CSF leakage in each treatment group (4\% in the miniplate and no plate treatment groups) (-Table 4).
- Wang et $\mathrm{al}^{9}$ reported that there were no spinal cord injuries or bleeding in either treatment group.

\section{Other Complications}

- One cardiopulmonary event was reported in the no plate treatment group (6\%) in one study (Jiang et al) $\left(-\right.$ Table 4). ${ }^{10}$

- Both studies (Jiang et al, ${ }^{10}$ Wang et $\mathrm{al}^{9}$ ) reported that there was no plate failure in any patient.

\section{Effect of Early Motion}

Both studies reported on postoperative care; however, none of the studies conducted a formal analysis of the effect of early cervical motion on postoperative axial pain. Jiang et al ${ }^{10}$ reported that patients in both treatment groups received the same postoperative care, which included the use of a cervical brace for 3 months after surgery; there was no significant difference in axial symptoms at final follow-up. Wang et $\mathrm{al}^{9}$ reported that the miniplate patients, who wore a collar for 2 weeks versus 6 weeks in the no plate group, experienced significantly less pain at 21.2 months as measured by the VAS score compared with the no plate patients, who wore a collar for 6 weeks (-Table 2).

\section{Evidence Summary}

The overall strength of evidence evaluating the comparative effectiveness of open door laminoplasty compared with 
Table 5 Evidence summary

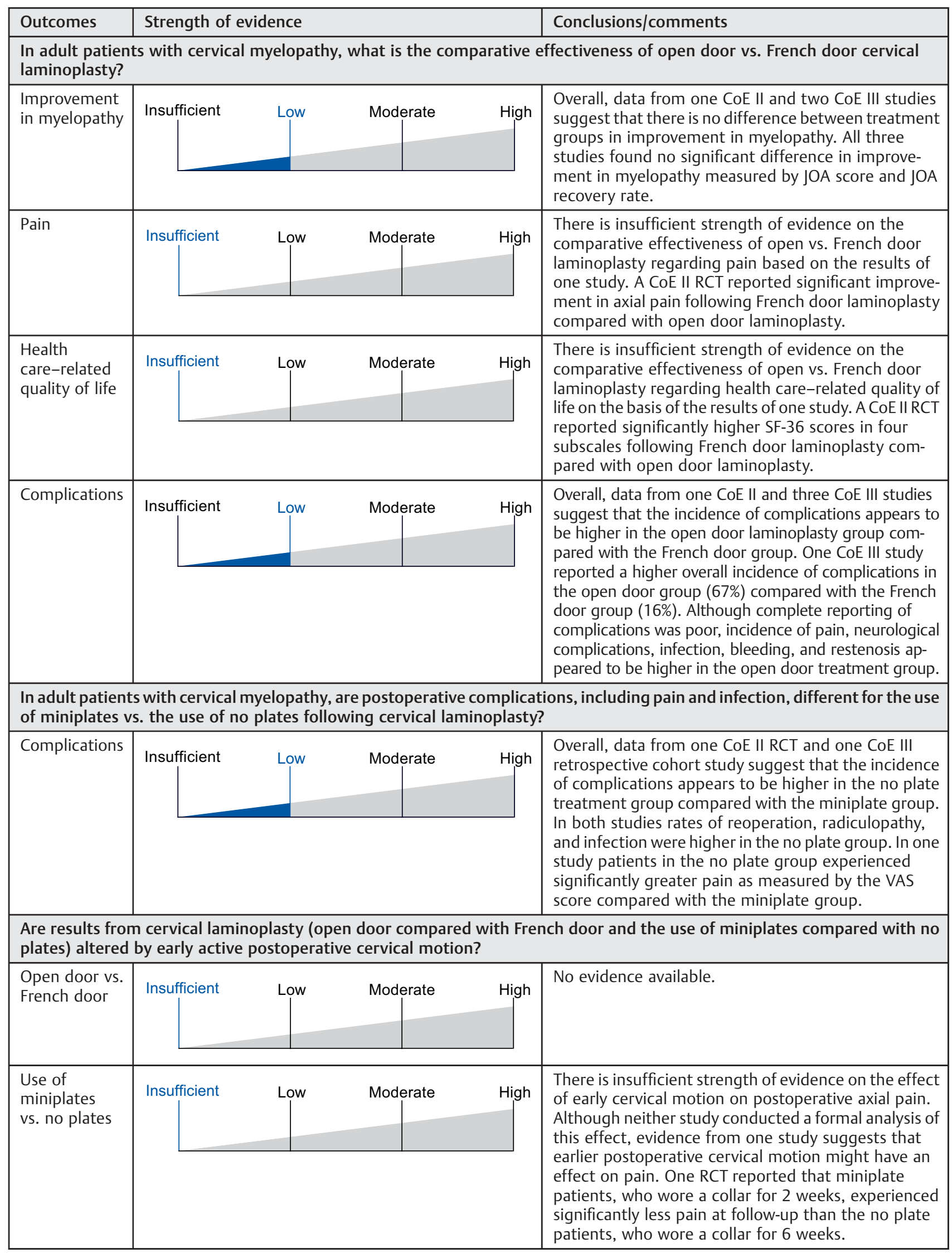

Abbreviations: CoE, class of evidence; JOA, Japanese Orthopedic Association score; RCT, randomized controlled trial; SF-36, short form 36; VAS, visual analog scale. 
French door laminoplasty regarding improvement in myelopathy and surgical complications is low, that is, there is a low confidence that the evidence reflects the true effect, and further research is likely to change the confidence in the estimate of effect and likely to change the estimate. The overall strength of evidence evaluating the comparative effectiveness of open door laminoplasty compared with French door laminoplasty regarding pain and health carerelated quality of life is insufficient, that is, the available evidence does not permit a conclusion. The overall strength of evidence evaluating the comparative effectiveness of the use of miniplates compared with the use of no plates in cervical laminoplasty regarding surgical complications is low, that is, there is a low confidence that the evidence reflects the true effect, and further research is likely to change the confidence in the estimate of effect and likely to change the estimate. The overall strength of evidence evaluating the effect of early cervical motion after open versus French door laminoplasty or the use of miniplates versus no plates after cervical laminoplasty is insufficient, that is, the available evidence does not permit a conclusion (-Table $\mathbf{5}$ ).

\section{Illustrative Case}

The patient is a 55-year-old male with myeloradiculopathy with minimal axial neck pain. He has congenital stenosis, OPLL, mild disc protrusions that efface the spinal cord from C4-5 down to C7-T1. He was treated with an open-door laminoplasty of $\mathrm{C} 5-7$ with miniplate fixation and partial laminectomy of C4. He was treated with early neck mobilization, non-steroidal anti-inflammatory drugs, and minimal narcotics. His preoperative symptoms completely resolved within several weeks postoperatively. He continued to do well at 2 years postoperatively (-Figs. $\mathbf{2 - 5}$ ).

\section{Discussion}

\section{- Limitations:}

- Few studies were available to address the KQs, all with small sample sizes $(n<86)$.

- Patient-reported outcomes, such as pain and SF-36, were reported in only one study for KQ1.

- Complete reporting of complications was poor; complications were commonly reported in only two studies.

- Despite the long history of use and the claimed relative advantages and disadvantages of open door laminoplasty versus French door laminoplasty, little comparative data exist, even in 2013. The best that one can glean from a structured review is that the data are "insufficient" to resolve the debate with respect to the superiority of either laminoplasty technique. This is largely because of the fact that laminoplasty evolved in an environment in which pupils tend to continue the practices passed down by their mentors.

- The theoretical advantages of rigid fixation of the laminae are intuitively appealing and have encouraged modifica-

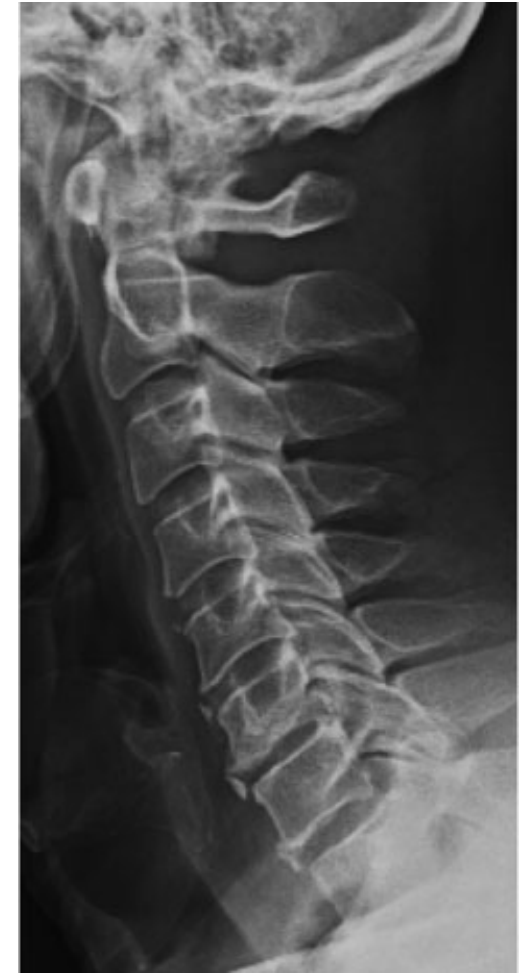

Fig. 2 Pre-op lateral radiograph.

tions in postoperative rehabilitation strategies. More data are needed to clearly establish what might be suggested by the available studies. Unfortunately, Jiang et al missed an opportunity to investigate the potential effect of early active range of motion when they still required the plated group to wear cervical collars for 3 months after surgery.

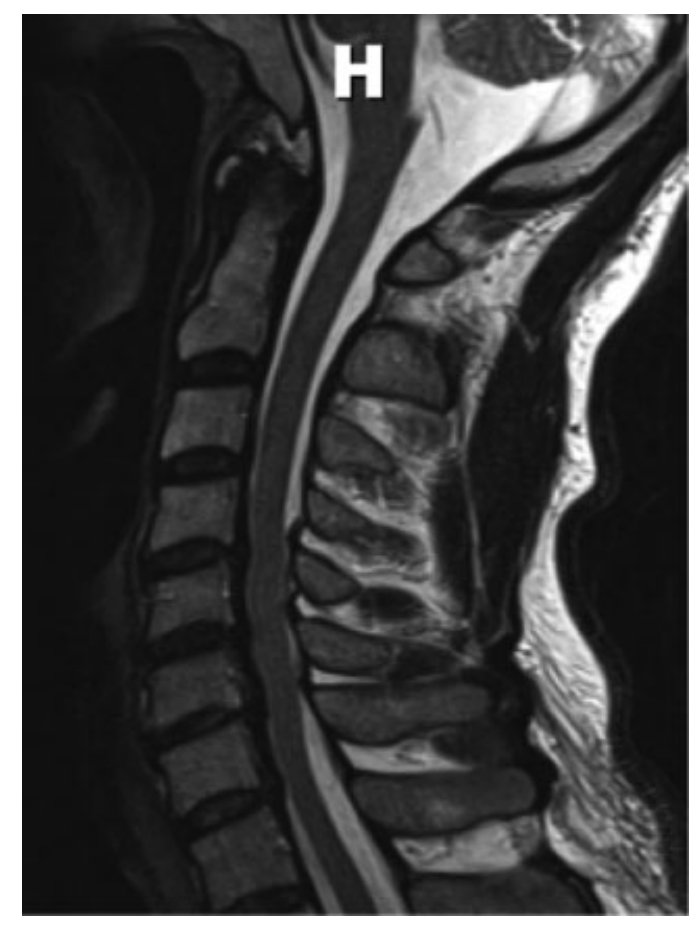

Fig. 3 Pre-op magnetic resonance image sagittal view. 


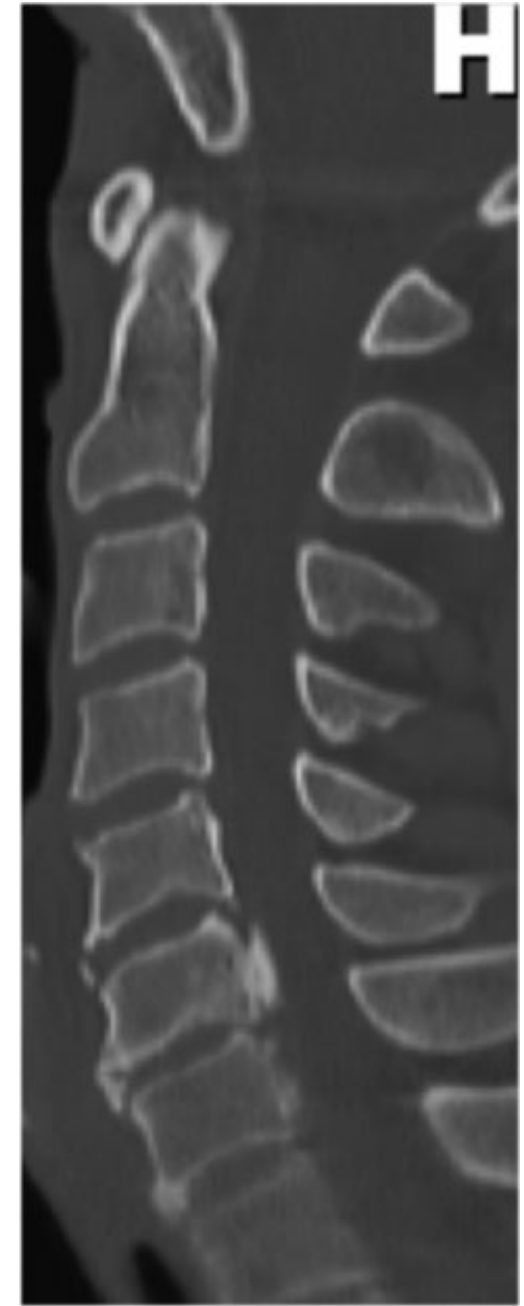

Fig. 4 Pre-op computed tomography sagittal view.

Wang et al came closer to shedding light on this issue when they used collars for only 2 weeks in their plated group versus their customary 6 weeks in the nonplated group. While the neurological outcomes were equivalent, there were significantly better SF-36 bodily pain, general health, and vitality scores in the plated group, which seemed to be primarily due to the increased postoperative axial pain among the no plate group.

- In the absence of either clinical equipoise or equal surgical facility at any given institution, it seems most reasonable to urge surgeon-scholars to pursue similarly constructed prospective clinical evaluations of a single option, while attempting to standardize the study methodologies among peer institutions engaged in alternative techniques so that the results can be analyzed appropriately. In this light, academic organizations may play an important role in unifying study design. With apologies to methodologic purists, perhaps we surgeons can then resolve an important and spirited debate.

\section{Summary and Conclusion}

Despite more than 30 years of clinical use and literature on laminoplasty, scant comparative studies exist. Data from

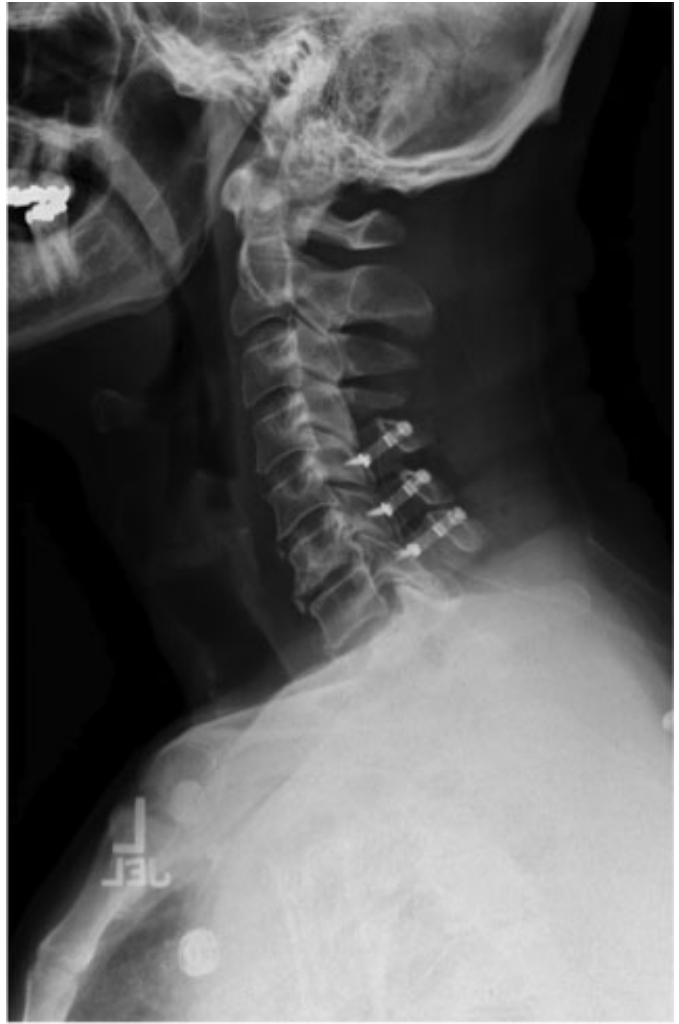

Fig. 5 Post-op lateral radiograph.

three such comparative studies are not sufficient to support the superiority of open door cervical laminoplasty or French door cervical laminoplasty. Data from two comparative studies are not sufficient to support the superiority of the use of miniplates or no plates following cervical laminoplasty. The overall strength of evidence to support any conclusions is low or insufficient. Thus, the debate continues while opportunity exists for the spine surgery community to resolve these issues with appropriately designed clinical studies.

\section{Conflict of Interest \\ None}

\section{References}

1 Hirabayashi K, Watanabe K, Wakano K, Suzuki N, Satomi K, Ishii Y. Expansive open-door laminoplasty for cervical spinal stenotic myelopathy. Spine 1983;8(7):693-699

2 Kurokawa T, Tsuyama N, Tanaka H. Double door laminaplasty through longitudinal splitting of the spinous processes for cervical myelopathy. Rinsho Seikei Geka 1984;19:483-490

3 Lehman RA Jr, Taylor BA, Rhee JM, Riew KD. Cervical laminaplasty. J Am Acad Orthop Surg 2008;16(1):47-56

4 Wright JG, Swiontkowski MF, Heckman JD. Introducing levels of evidence to the journal. J Bone Joint Surg Am 2003;85-A(1):1-3

5 Atkins D, Best D, Briss PA, et al; GRADE Working Group. Grading quality of evidence and strength of recommendations. BMJ 2004; 328(7454):1490

6 Okada M, Minamide A, Endo T, et al. A prospective randomized study of clinical outcomes in patients with cervical compressive myelopathy treated with open-door or French-door laminoplasty. Spine 2009;34(11):1119-1126 
7 Yue WM, Tan CT, Tan SB, Tan SK, Tay BK. Results of cervical laminoplasty and a comparison between single and double trapdoor techniques. J Spinal Disord 2000;13(4):329-335

8 Naito M, Ogata K, Kurose S, Oyama M. Canal-expansive laminoplasty in 83 patients with cervical myelopathy. A comparative study of three different procedures. Int Orthop 1994;18(6):347-351
9 Wang L, Song Y, Liu L, et al. Clinical outcomes of two different types of open-door laminoplasties for cervical compressive myelopathy: a prospective study. Neurol India 2012;60(2):210-216

10 Jiang L, Chen W, Chen Q, Xu K, Wu Q, Li F. Clinical application of a new plate fixation system in open-door laminoplasty. Orthopedics 2012;35(2):e225-e231

\section{Editorial Perspective}

The two authors of this systematic review on laminoplasty can be rightly considered to be key opinion leaders on this topic and have provided us with the most comprehensive overview of some fundamental questions regarding basic technique variations-a long overdue effort. From the 50 or more different laminoplasty techniques, it is traditions and anecdotally based beliefs that have governed clinical decision-making more than actual evidence-based guidance. It comes as little surprise that at the present time, our evidence base unfortunately offers few clear treatment recommendations. With these results to date, surgeon preference and patient factors (such as size of posterior spinal elements) remain relevant decision-making variables. From the published data, it appears also reasonable to assume that early range of motion with stable fixation of expanded arches is a safe and effective treatment strategy, and that prolonged postoperative immobilization is not conducive to attaining better outcomes. For the more specific technique-related questions, there is ample room for future investigations. Drs. Heller and Riew have provided the spine community a solid platform from which to move forward from. 